\title{
Implementing a Score-Time based Model for Handling Deadlocks in Football Group Matches
}

\author{
Olusola Olajide Ajayi \\ Department of Computer Science, Faculty of Science, \\ Adekunle Ajasin University, Akungba-Akoko, Ondo State, Nigeria
}

\begin{abstract}
In a typical football event, three possibilities can occur: a winner, a loser/runners-up, or a tie. The latter however is not left in that status especially when it has to do with a group or league game; as a resolve is needed to establish two partitions - winner and loser. Different deadlock models are employed to resolve different deadlock scenarios. Football histories and events have however shown that most models failed to solve some complex deadlocks. Tossing of coin is a particular kind of model that starts where others stopped. Taking the last Africa Cup of Nations (AFCON) 2015 Football Fiesta as a case study however, the use of the 'last man standing' deadlock model - coin tossing, proved unpleasant to both teams in questions and generated lots of heat from football fans of both countries. Football at this age has indeed proved in so many ways to be technological-oriented. Bringing manual 'decision system' into a game of this century and magnitude prompted the researcher to present an ideal and implementable model that resolves deadlock situations in football group matches.
\end{abstract}

\section{Keyword}

Football, deadlock, model, coin tossing, AFCON, game, group matches

\section{INTRODUCTION}

The game of soccer can be describe as a game involving two teams, each at playing from one end of the field towards the opponent's side. Each team s made up of eleven (11) players with some number of reserve players, each playing/defending from opposite side of the field with a goal post at both ends. It can also be refer to as a 'round-leather' game consisting of two teams vying for victory over each other. A team is said to have scored the other when she succeeded in kicking the ball across the goal line of the other opponent. It can be regarded as a game that does not only requires technicality but skills and has produced the likes of the legend Pele of Brazil to the great Abedi Pele of Ghana, the magical Diego Armando Maradona of Argentina to the skillful Austin Okocha of Nigeria.

The contemporary history of the world's favourite game spans more than 100 years. It all began in 1863 in England, when rugby football and association football branched off on their different courses and the Football Association in England was formed - becoming the sport's first governing body.

Both codes stemmed from a common root and both have a long and intricately branched ancestral tree. A search down the centuries reveals at least half a dozen different games, varying to different degrees, and to which the historical development of football has been traced back. Whether this can be justified in some instances is disputable. Nevertheless, the fact remains that people have enjoyed kicking a ball about for thousands of years and there is absolutely no reason to consider it an aberration of the more 'natural' form of playing a ball with the hands.

On the contrary, apart from the need to employ the legs and feet in tough tussles for the ball, often without any laws for protection, it was recognised right at the outset that the art of controlling the ball with the feet was not easy and, as such, required no small measure of skill. The very earliest form of the game for which there is scientific evidence was an exercise from a military manual dating back to the second and third centuries BC in China.

This Han Dynasty forebear of football was called Tsu' Chu and it consisted of kicking a leather ball filled with feathers and hair through an opening, measuring only $30-40 \mathrm{~cm}$ in width, into a small net fixed onto long bamboo canes. According to one variation of this exercise, the player was not permitted to aim at his target unimpeded, but had to use his feet, chest, back and shoulders while trying to withstand the attacks of his opponents. Use of the hands was not permitted.

Another form of the game, also originating from the Far East, was the Japanese Kemari, which began some 500-600 years later and is still played today. This is a sport lacking the competitive element of Tsu' Chu with no struggle for possession involved. Standing in a circle, the players had to pass the ball to each other, in a relatively small space, trying not to let it touch the ground.

The Greek 'Episkyros' - of which few concrete details survive - was much livelier, as was the Roman 'Harpastum'. The latter was played out with a smaller ball by two teams on a rectangular field marked by boundary lines and a centre line. The objective was to get the ball over the opposition's boundary lines and as players passed it between themselves, trickery was the order of the day. The game remained popular for 700-800 years, but, although the Romans took it to Britain with them, the use of feet was so small as to scarcely be of consequence.

While the sport was widely played by youngsters all over England, it was banned in schools because of its fierceness. Lack of rules governing player conduct often resulted in injuries and chaos. That is why in the 19th Century, clubs felt the urge of creating rules. So they gathered together and formed the Football Association.

Since then, the association has metamorphosed into what is today called Federation of International Football Association (FIFA). FIFA has been served by eight Presidents since its foundation in 1904; from Frenchman Robert Guerin to the current incumbent Joseph S. Blatter. Each President has played his part in the growth and development of the world's favourite game.

Since the inception of its global competition (FIFA World Cup) in Uruguay, 1930, no other sporting event has increased 
so much in popularity and prestige and became the cynosure of the whole world, like the game of football.

One way by which transformations and innovations has been brought to the game is the introduction of rules. The seventeen (17) laws formulated by International Football Association Board (IFAB) range from rules pertaining to the game of play, to the rules governing corner kick. Aside these rules that promote fair play, there are also rules that pertain to resolving deadlocks in matches or group matches. In sport, a 'deadlock' means a drawn game, a tie, when both sides have the same score (in football, usually 0-0). It is worth stating here however, that, there are varying deadlock situations in the game of soccer, leading to the use of a particular type of deadlock model. Having examined the limitations of some of the existing deadlock models; including extra-time, suddendeath, penalty shoot-out, re-match, aggregate point difference, and die-casting/coin tossing, this paper seeks to introduce the use of score-time-based deadlock model in addressing complex deadlocks in football group matches.

\section{STATEMENT OF PROBLEM}

Most complex deadlocks in group or league soccer games are usually resolved using home-and-away score model; where the score values of the two tied teams are taken into consideration for both their home and away matches. Where this model did not work, the football management resolves to adopt the aggregate point difference model; where the goal forward and goal against figures are taken into consideration. Notable however is a situation whereby these two previous models failed to break the 'yoke'. The question is: where these technical models failed to resolve the deadlock, what other option is left? Inability to provide a technical solution made the management to settle for a non-technical solution coin tossing (www.allsports.com.gh).

This was the ugly situation that presented itself at the just concluded AFCON 2015 Group D game; where Guinea and Mali were tied on points and goal difference.

Using the AFCON 2015 Soccer Competition and citing GROUP D (consisting of Ivory Coast, Cameroon, Guinea and Mali) as a Case Study, where all the teams were tied as at the end of the $2^{\text {nd }}$ matches. Going into the $3^{\text {rd }}$ matches, the rules/permutations for selecting the two (2) qualifying teams are: (deadlock situations and their resolves)

i) If the two teams on one side played goalless draw (i.e. 0-0), while the other two teams played goaldraw (i.e. $1-1$ or $2-2$ etc), these two (2) teams will go through.

ii) If the two teams from both matches played goaldraw (i.e. $1-1$ or $2-2$ etc), the two teams with the highest goal scored will go through.

iii) If all the teams played out draw, with the same goal margin, then coin will be tossed to choose who qualifies.

iv) If in one of the matches, there was an eventual winner, but the other two teams played out a draw (either goal-draw or goalless draw), then a coin is tossed to choose the second $\left(2^{\text {nd }}\right)$ qualifying team among the teams that played draw.

The question is: Why will semi-manual process be brought in? Why tossing coin at this technological age? The adoption of the coin tossing deadlock model was adjudged inappropriate (www.sports.yahoo.com).
This article therefore intends displacing this non-technical approach by presenting a computing model that helps resolve such complex deadlock.

\section{MOTIVATION}

Occurrence of deadlock at the just concluded AFCON 2015 Football Competition between Guinea and Mali (in Group D, alongside Ivory Coast and Cameroon) serves as the motivating ground for this work. The negative reactions that sprang up, not only from the Malians, but also from football loving fans, prompted the need to research into alternative, better, and systematic approach to resolving deadlock of such kind.

\section{METHODOLOGY}

This study shall adopt the following methodologies:

i. Phase 1 (Model Design): Score-time-based model, with appropriate algorithms was adopted. The Unified Modelling Language (UML) was used.

ii. Phase 2 (Model Implementation): An objectoriented approach was adopted for the implementation of the proposed model.

iii. Phase 3 (Model Demonstration): Java programming technique was deployed to achieve the implementation of the model.

\section{REVIEWED LITERATURE}

From the literatures gathered, the following research findings were made on the existing football deadlock resolve methods/models:

(i) Home-and-Away Scores: This model is mostly used for tournament's qualifying matches. In it, a tie is resolved by considering the goal scored by both teams on home and away basis. For instance, if Teams A and B played 1-1 (at Team A's home) and played 0-0 at Team B's ground, it is adjudged that, Team B goes through on home-and-away goal rule; as Team B managed to score more in Team A's home, while Team A scored less in Team B's home.

Algorithm: The algorithm looks thus:

While (game tie at home and away games)

$$
\begin{aligned}
& \text { If }(\mathrm{t} 1 \text { _home goal > } \mathrm{t} 1 \text { away_goal }) \text {.AND. } \\
& \text { (t2_away_goal > t2_home_goal) .AND } \\
& \text { (t1_away_goal !> t2_home_goal). AND. } \\
& \text { If }(\mathrm{t} 2 \text { _home_goal }==\mathrm{t} 1 \text { _away_goal .AND } \\
& \text { t1_away_goal ! t2_away_goal) } \\
& \text { t2 emerges winner } \\
& \text { else if(t1_home_goal < t1_away_goal) .AND } \\
& \text { (t2_away_goal < t2_home_goal) .AND. } \\
& \text { (t2_home_goal !> t1_away_goal) .AND. } \\
& \text { if }(\mathrm{t} 2 \text { _home_goal == t1_away_goal .AND. } \\
& \text { t2_away_goal !> t1_away_goal) } \\
& \text { t1 emerges winner } \\
& \text { else } \\
& \text { tie yet unbroken } \\
& \text { resort to any other model (i.e. extra } \\
& \text { time/sudden death) }
\end{aligned}
$$

end while

(ii) Aggregate Score: Where the home-and-away score rule results into tie, the next available model to be considered is the aggregate rule. In this model, the number of goals scored 
(GF) and the number of goals scored against (GA) for both teams are compared. For instance, if Team A has more GF than Team B, Team A goes through, else Team B. in instances where their GF is the same, the tem with the less GA is adjudged the winner, else we resort into the next model.

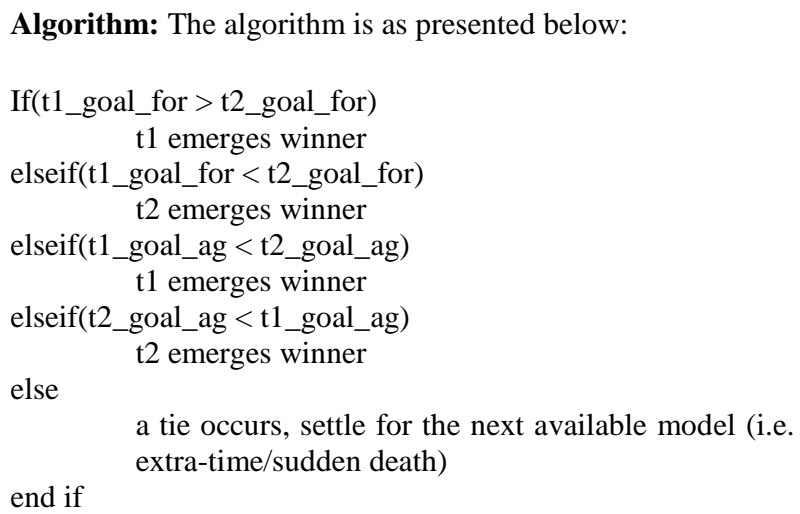

(iii) Extra-Time/Sudden death: We resort to this model where the tie is still somehow 'tight' to break. In other words, we bring in this model when after regulation time and the deployment of home-and-away score rule could not resolve the tie. In this model, extra 30minutes of play (15minutes for each half) is allowed. During this time, any team that scores first is taking as the winner of the game. In case of tie, the next model (penalty shoot-out) is then considered.

Algorithm: The algorithm goes thus:

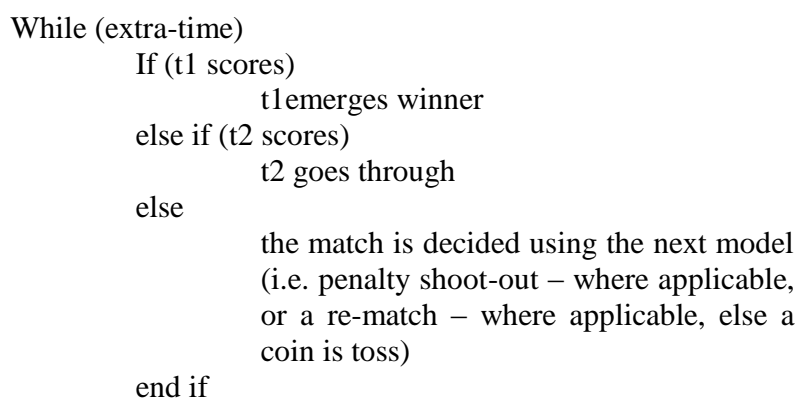
end while

(iv) Penalty Shoot-out: This type of deadlock resolve is brought into play where two teams played out either a barren draw (i.e. $0-0$ ) or score draw (i.e. 1-1, 2-2 etc.). It is mostly used outside the group matches (i.e. during knock-out stage, quarter final, semi-final, and/or final). In this model, each team presents five(5) players for the shoot-out. If after the shot out, the standing remains tie, the shot is turned into sudden death, that is, each team presents a player each; in which the shooting continues until a player looses a kick and the other scores. If the tie continues and all the eleven (11) players have had their kicks, then each team's goalkeeper is permitted to kick for his team. If still ties, the pointer is reset back to the players until a winner emerges.

Algorithm: The algorithm below describes the above highlighted model.

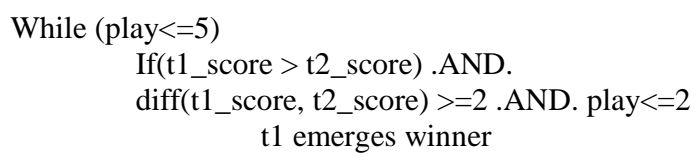
else
shoot out continues until a team loses and the other scores
end if

end while

(v) Re-match: Where possible (i.e. during qualifying group matches), this model is adopted when other previous deadlock resolve models have failed. In this, a re-play (re-match) of the game is scheduled for a different date but perhaps, same venue. The pointer is then reset to some of the earlier models, depending on the outcome of the re-match, to determine the winner. But where ties, coin tossing is taken as the last resort.

Algorithm: The logical procedure is described thus:

While (other previous models failed to resolve deadlock) Stage a re-match (reset other appropriate models i.e. extra time/sudden death, penalty shoot-out etc) If(re-match traps a winner) report the winner else end if resort to coin tossing

end while

(vi) Coin Tossing: This model uses random number output to determine a winner in a deadlock. In this, a coin (head or tail, win or loss, 1 or 0 ) represents a pointer for each team. In the case of our case study (AFCON 2015 deadlock between Guinea and Mali), two balls with position two (2) and position three (3) were presented (as the coin with two options) in a pot, and the representative of the two teams was to pick a ball from the pot. Whoever picks the ball in position two (2) emerges as the winner/qualifier, else a loser (africanfootball.com). Unfortunately, Mali picked the ball in pot three (3), while Guinea picked the ball in pot two (2) and goes through as the runners up in the group (after Ivory Coast).

Algorithm: The algorithm for this random selection is:

While (two balls in the pot) If(t1 picks a ball in pos2) t1 breaks the deadlock \& goes thru else t2 breaks the deadlock \& goes thru

end while

\section{end if}




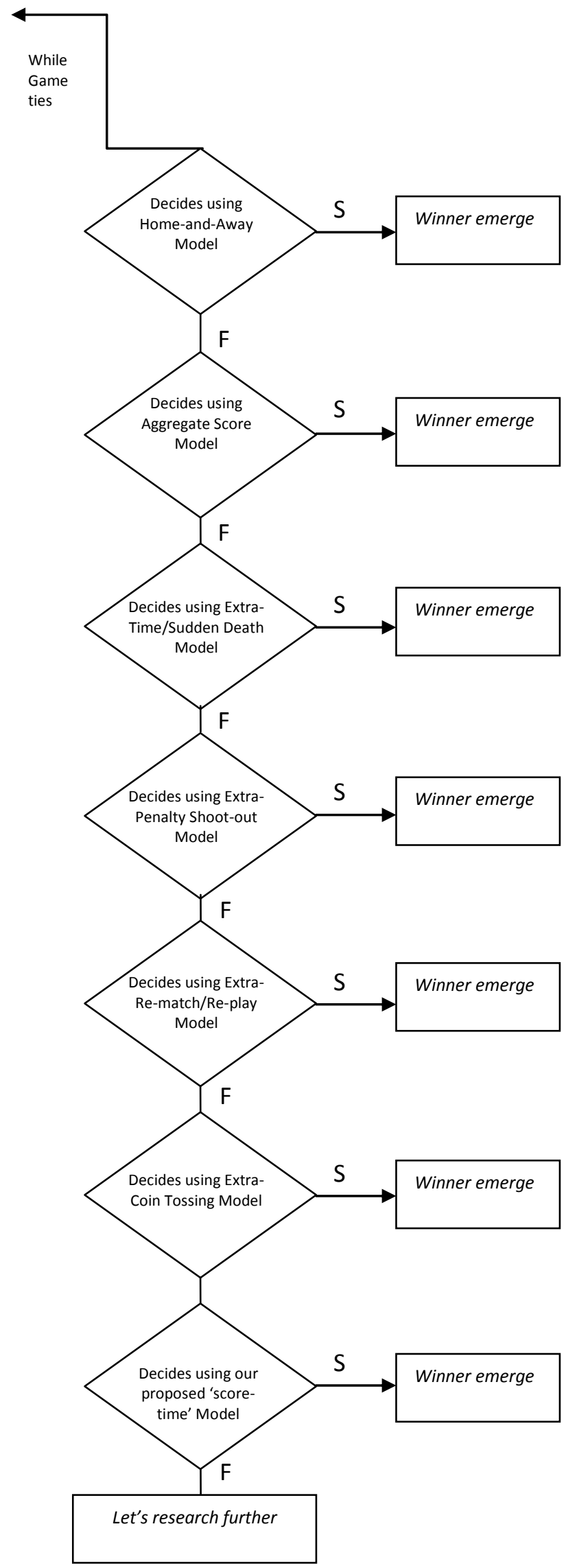

Fig.1: Existing deadlock models' flow

\section{THE PROPOSED DEADLOCK MODEL}

The proposed deadlock resolve model make use of score-time of each team in conflict as it relates to their previous matches with other teams.

Algorithm: The proposed model algorithm is as shown below:

While (deadlock exists)

For (i) (as many matches played)

$\begin{aligned} & \text { fetch (t1_score_time against } \\ & \text { other_team }(\mathrm{n}-(\mathrm{n}-\mathrm{i}))\end{aligned}$
sum up t1_score_time
$\begin{aligned} & \text { fetch (t2_score_time } \\ & \text { other_team(n-(n-i)) }\end{aligned}$
sum up t2_score_time

// $\mathrm{n}$ implies number of teams

continue loop (increment no of matches)

end loop

//compare score_time

if(t1_score_time > t2_score_time)

t1 goes thru

elseif(t1_score_time $<$ t2_score_time)

t2 goes thru

else

deadlock remains unresolved (adopts hybrid deadlock resolve model e.g. no of goals attempt)

end if

end while 


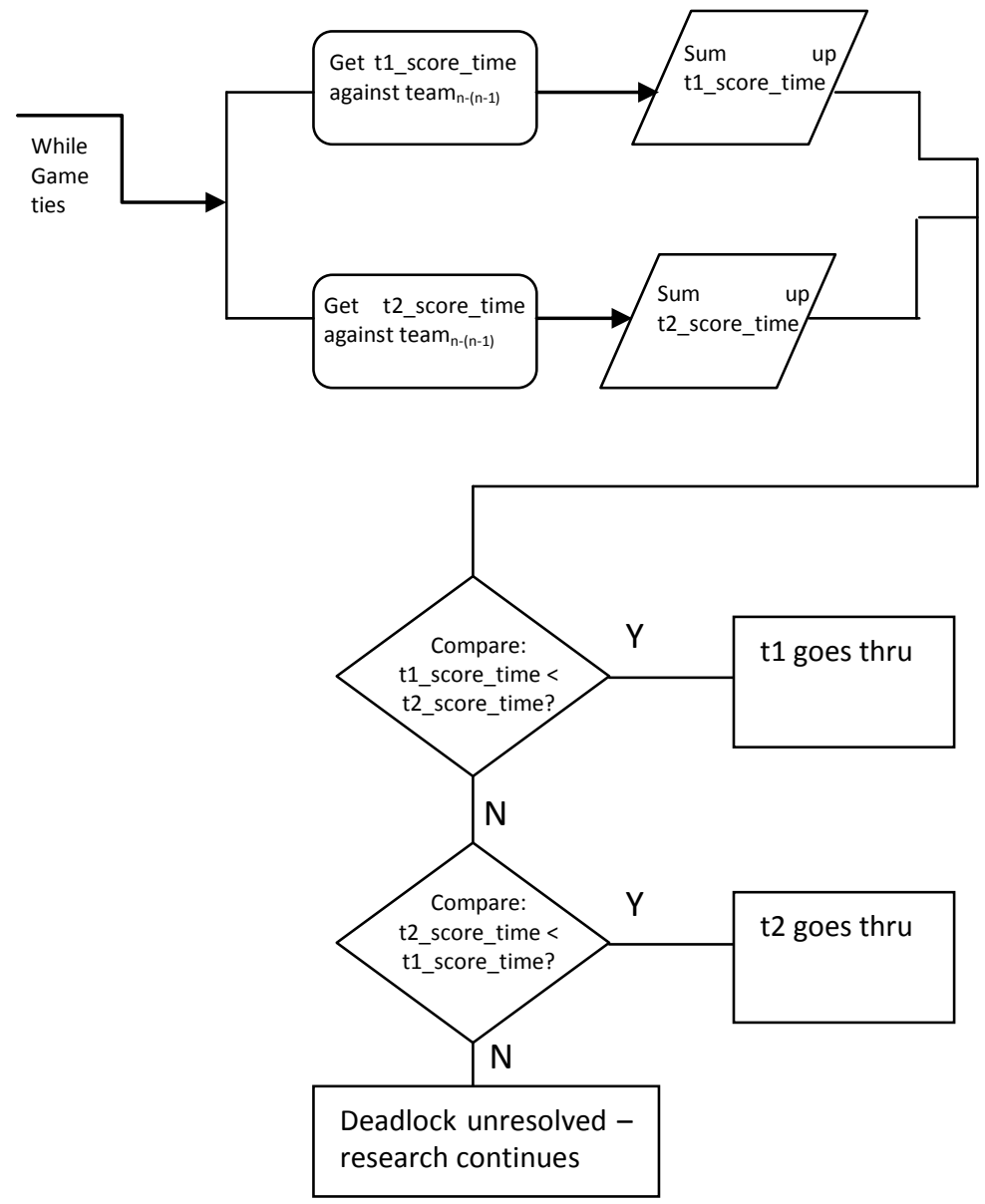

Fig. 2: The proposed model's flow

\section{MODEL IMPLEMENTATION}

The model was implemented using JAVA GUI and Array facilities based on the described proposed algorithm/computational model.

\section{CONCLUSION}

Logically, this paper has been able to establish the need to remodel the existing models of resolving deadlock in football soccer match. Though lacking the needed link for controlling all-round goalless deadlock situation, the article has to some extent; argue for the need to phase out the coin tossing method of deciding deadlock in football group matches.

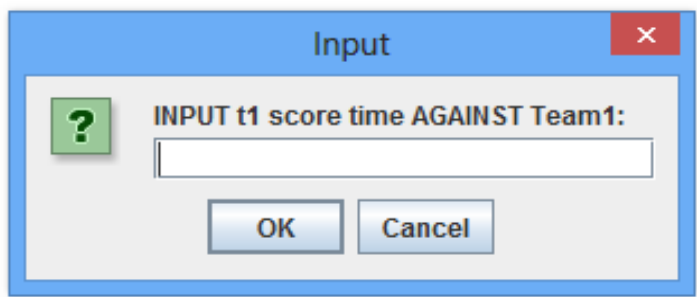

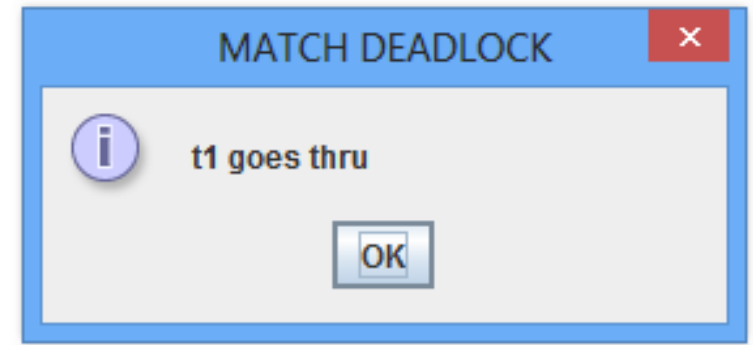

\section{SUGGESTION FOR WORK IMPROVEMENT}

The major limitation of the proposed model is that it might not work for a situation whereby the teams in question all played goal-less draw in all their matches (as the name of the model suggests - score-time). However the work can be bettered by employing an hybrid method, inculcating goal attempts. This will also bring about a kind of improvement into the game; as teams will now tend to playing more of attacking and entertaining football than defensive play - as Jose Mourinho (Chelsea Coach) will usually do in a tight match (www.zonalmarking.net).

\section{REFERENCES}

[1] FIFA (2014). Laws of the Game. FIFA-Strasse 20, 8044 Zurich, Switzerland.

[2] D.-S. Huang, L. Heutte, and M. Loog (2007): Fuzzy Decision Method for Motion Deadlock Resolving in Robot Soccer Games. ICIC 2007, LNCS 4681, pp. 1337 1346, Springer-Verlag Berlin Heidelberg.

[3] www.fifa.com. Accessed 02/02/2015

[4] http://sports.yahoo.com/news/guinea-mali-drawing-lotscup-nations-201106356--sow.html. Accessed 13/03/2015

[5] http://allsports.com.gh/football/teams/blackstars/deadlock-draw-of-lot-to-determine-mali-or-guineaas-next-ghana-opponents-id3442806.html). Accessed $13 / 03 / 2015$

[6] http://africanfootball.com/news/503152/How-Guinea-vsMali-toss-of-coin-will-be-done. Accessed 15/03/2015

[7] http://www.bbc.com/sport/0/football/27092978. Accessed 15/03/2015

[8] http://www.zonalmarking.net/2014/04/28/liverpool-0-2chelsea-chelsea-play-ultra-defensively-but-win-thegame/ Accessed 15/03/2015 